\title{
Review and Prospect of Emergency Logistics under Uncertainty Conditions
}

\author{
Hanping Zhao ${ }^{1,2 *}$, Chence Niu ${ }^{1,2}$, Tingting Zhang ${ }^{1,2}$, Sida Cai ${ }^{1,2}$ \\ ${ }^{1}$ Key Laboratory of Environmental Change and Natural Disaster, Ministry of Education of China, Beijing Normal \\ University, Beijing 100875, China \\ ${ }^{2}$ Academy of Disaster Reduction and Emergency Management, Ministry of Civil Affairs \& Ministry of Education \\ the Peoples' Republic of China, Beijing Normal University, Beijing 100875, China
}

Received March 21, 2018

Accepted April 20, 2018

\begin{abstract}
Emergency logistics is the key process of disaster relief activities. Abruptness and inaccurate prediction of disaster make emergency logistics operated under the condition of uncertainty; these uncertainties are essentially different from ones in traditional business decision. To seek the core and future of research in emergency logistics optimization, the mainly research of emergency logistics under uncertainty conditions in recent years were reviewed. The characteristics of uncertainty appearing in emergency logistics process were analyzed, and the expression of uncertain factors, demand forecasts and objective function definition in decision-making optimization model were summarized; moreover, this article analyzed the key points of research in aspects of emergency logistics operations, uncertainties origination and emergency risks, and provided useful reference and directions for future research.
\end{abstract}

Keywords: emergency logistics, uncertainty, demand forecasts, optimization

\section{不确定条件下的应急物流研究综述与展望 赵晗萍 ${ }^{1,2^{*}}$, 牛晨策 ${ }^{1,2}$, 张婷婷 ${ }^{1,2}$, 蔡思达 ${ }^{1,2}$}

1. 北京师范大学环境演变与自然灾害教育部重点实验室, 北京 100875

2. 北京师范大学，民政部/教育部减灾与应急管理研究院，北京 100875

\begin{abstract}
摘 要: 突发事件发生具有突发性和无法准确预测性的特点, 这导致应急物流在构建与运作过程中存在不 确定性。这种不确定性与传统商业决策问题中的不确定性有着本质差别。为了确定应急物流研究的核心和 方向, 本文对近些年来国内外不确定条件下应急物流优化方面的研究进行了综述：从灾害的本身特点出 发，阐述了应急物流问题的特征；从不确定性因素的刻画、需求预测与决策目标定义三个方面总结应急物 流主要研究进展; 最后, 本文建议, 应该将应急物流运作、不确定因素的来源和应急过程风险评估等三个 方向, 作为应急物流问题的研究重点。
\end{abstract}

关键词: 应急物流, 不确定性, 需求预测, 优化

应急物流是为了满足紧急情况下受灾人员的应 急需求, 从救灾点向突发事件发生地, 对应急物

作者简介: 赵晗萍 (1977-), 女, 汉, 副教授, 博士, 研究方向: 灾害风险评价、离散系统仿真、应急响应技术 资助项目: 国家自然科学基金(41471424); 中央高校基本 科研业务费专项资金资助
资、信息以及服务的有效流动进行计划、管理与控 制的过程 ${ }^{[1]}$ 。它是应急响应的核心活动, 它能否有 效的运作是保证灾后幸存者维持生命和生活的关键 [2]。应急物流要根据灾情或灾害风险评估结果来确 定救灾物资的需求，然后根据需求信息安排物资的 供应，从各级物资储备库或潜在物资的供应商调运 到受灾地点, 发放到物资需求者的手中。 
国内外对应急物流问题越来越关注，一些学者 对应急物流方面研究做了详尽综述。如, Kovacs 等 ${ }^{[3]}$ 对灾害救助中的物流运营方面做了详细综述; 陈 丽群 ${ }^{[4]}$ 对应急物流中有关物资调运的研究进行分析 总结; 李创 ${ }^{[5]}$ 归纳整理了近些年国内外应急物流的 研究文献, 并从基础理论、物资调运和运输优化、 系统构建三个方面入手, 对国内外应急物流的研究 现状进行了综述分析。高文军等 ${ }^{[6]}$ 从基本理论、系 统构建、风险、存在问题、存在问题对策及相关模 型六个方面对应急物流的研究进展进行了综述; Galindo 和 $\mathrm{Batt}^{[\mathrm{f]}}$ 在 2013 年从灾害管理中的运用方 法、参与环节、灾害的类型、研究贡献和研究过程 中的假设这几个方面对灾害管理研究现状进行综述 分析。目前, 应急物流决策优化问题的研究是从传 统运筹学模型改进与扩展而来, 其主要分类如图 1 所示。

\section{1. 应急物流问题特征分析}

\section{1 不完备的需求信息}

自然灾害的发生难以精确预测，不具有周期 性, 加之人类行为活动的不可预测性, 应急物流的 需求不确定性的复杂程度要高于传统物流问题。灾 害发生后短期灾情速报信息通常是不完备不准确 的。如，2014年云南“8.03”鲁甸地震由于偏远地区村 寨在地震中有大量山体垮塌, 部分村寨几乎整村被 掩埋, 救援力量到达后才得以进行详细统计。8月3 日当日统计的死亡人数为 221 人, 但到了 8 月 7 日截 止到 19:00时的死亡人数已经达到了 615人, 灾情信 息在短时间内发生意料之外的波动。此外, 需求信 息的发布者并不是物资的需求人一一通常灾害救助 的政府管理者或者慈善团体才是发布需求信息的主

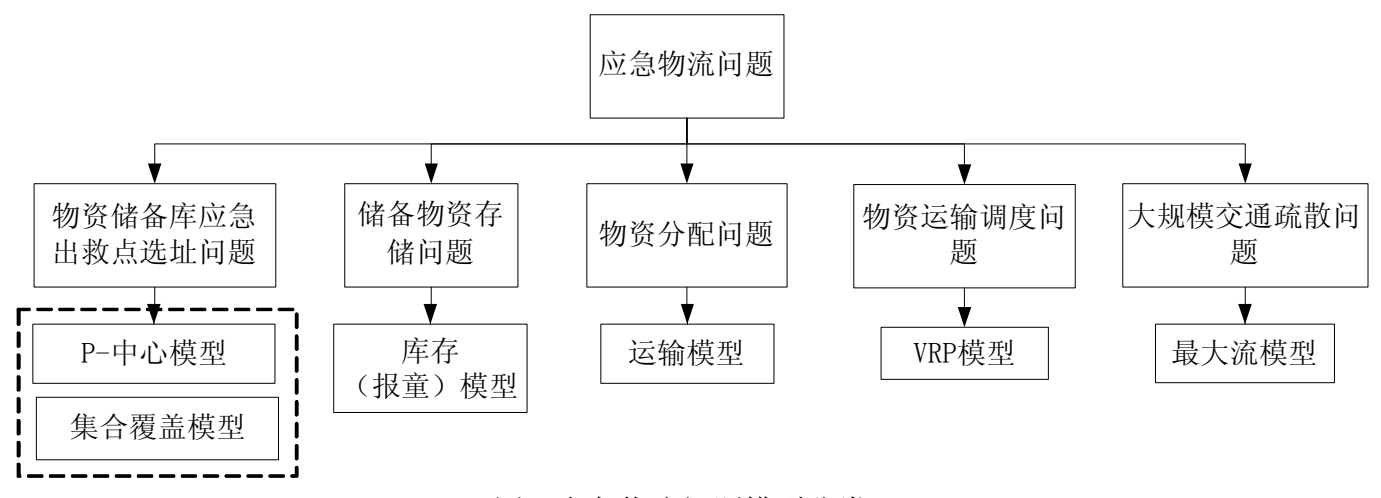

图 1 应急物流问题模型分类

但应急物流问题研究必须要正视其自身的属性 特征, 在突发事件的应急响应过程中, 面对的往往 是个不可预测极端不确定的环境, 应急救助过程中 常常发生各种极端干扰 (公路网条件差、内部冲突 和有限的技术资源等）而导致应急供应链无法正常 运行甚至中断, 发展中国家的灾害管理面临的这一 问题更加显著。BBC（2011年）的报告, 在最近十 年遭受自然灾害影响的 27 亿人中, 有 $99 \%$ 的人生活 在发展中国家 ${ }^{[8]}$ 。在2008年5.12汶川地震发生后, 途 经重灾区的G317和G213道路中断, 导致无法获取灾 情信息、救援力量无法进入灾区等应急响应活动受 阻。

不确定性是应急管理中必须要重点关注的因 素, 解决应急物流问题也要从本质上分析其不确定 特征、刻画不确定因素、解决需求预测问题、合理 定义应急决策目标。因此本文将在分析应急物流不 确定因素特点基础上, 总结应急物流研究进展并展 望未来主要研究方向。
体; 需求信息的发布存在不确定性一一需求的信息 在各级物资调配部门之间传递过程中, 会由于各种 原因导致需求信息的进一步变异或失真。

\section{2 多样化的不确定因素}

应急物流系统不是一个稳定运行的日常系统， 不仅是需求难以预测, 其供应的主体是由很多渠道 组成的, 包括政府、非政府组织、企业、个人等。 在应急状态下对物资的供应并不是按照需求来供 给, 其中会有诸多因素 (如紧迫性、媒体與论等) 来影响不同受灾区域的供给数量。在物资调运中会 有意外干扰毁坏交通路网和信息通讯导致供应中断 或延迟、甚至在有的极端情况下提前准备好的库存 会在灾害发生后被毁于一旦, 这使得应急物资在生 产和运输过程中也会发生不确定变化。因此应急物 流系统中不确定因素种类是多样的。应急物流问题 也会因致灾因子、需求物资类型、应急过程不同而 
存在本质差异, 比如不可预测地震和可以预测的台 风, 前者侧重于灾后响应后者侧重于灾前备灾, 前 者对于需求无法预报后者可以进行概率预报。因此 针对不同应急情景和问题要选取恰当不确定因素描 述方式。

\section{3 非逐利的决策目标}

应急物流不单追求在物资成本、运输成本、库 存成本等直接成本的最小化, 而是更关注在应急过 程中如何保证公众安全, 如由于物资短缺而产生的 人员伤亡损失，物资公平性分配的满意度评价等。 因此如何选取决策目标的评价指标是一个关键问 题。应急决策过程中会出现各种不确定条件甚至是 极端事件（低概率高损失）干扰，那么如何建立一 个合理目标度量方式, 使得决策方案在发生各种不 确定变化情况下依然稳健也是需要关注的问题。

针对考虑不确定性因素与干扰条件下的应急物 流决策优化问题的研究，表 1 将主要文献进行分类 汇总。在第 2 节中, 将具体介绍其研究方法。

\section{2. 不确定应急物流问题处理方法}

一个考虑不确定性应急优化问题基本表达形式 如下:

$$
\begin{aligned}
& \min / \max \quad F(x, b, \zeta) \\
& \text { s.t. } g_{j}(x, \zeta) \leq 0, \quad j=1,2, \cdots, J
\end{aligned}
$$

其中, $\zeta$ 为不确定性参数, $x$ 为决策向量, $b$ 为参数 向量; $F(x, b, \zeta)$ 为目标函数, 表达了衡量应急物流 运行效率的标准, 如成本、时间、需求被满足程度 等; $g_{j}(x, \zeta) \leq 0$ 为约束条件函数, 通常应急过程中 受到人力、财力、时间、物资的限制, 还会受到交 通网络、决策时间逻辑等条件约束。对于包含不确 定因素的应急物流决策问题, 首先要找到适合于模 型中的不确定性因素的刻画方法, 然后确定合适的 决策目标, 并用有效的算法求解这个问题。

\section{1 不确定因素刻画方法}

目前应急物流优化问题中，描述不确定性主要 有以下几种的方法:

(1) 区间不确定集合

式(1)中不确定因素 $\zeta$ 可看作一个对称有界的随 机变量, 在对称区间 $\left(\zeta^{0}-\zeta, \zeta^{0}+\zeta\right)$ 内取值, 即区 间型不确定集合

$$
\Xi=\left\{\zeta \mid \zeta \in\left(\zeta^{0}-\zeta, \zeta^{0}+\zeta\right)\right\}
$$

其中 $\zeta^{0}$ 为区间的均值， $\zeta$ 则定义了区间的边界。

应用区间不确定集合估计不确定性比较粗糙, 但可以方便的转化成为线性规划模型, 目前有一些 学者 ${ }^{[9-11]}$ 利用这种方法估计应急需求, 并采用鲁棒优 化技术解决该问题。

（2）情景分析法

情景分析法将利用构成所研究的系统问题的关 键事件（因素）及每个事件和事件之间的发展变化 的概率（影响程度）, 对事件未来的发展状态或结 果进行分析与描述 ${ }^{[39]}$ 。按照灾害等级或者影响程度 将灾后划分为不同情景 $\omega, \omega=1,2, \ldots, s$, 并定义情景 $\omega$ 发生概率为 $p(\omega)$, 且 $\sum_{\omega=1}^{s} p(\omega)=1$ 。用 $\zeta_{\omega}$ 记在情 景 $\omega$ 中不确定性因素向量。

情景分析法是目前应急问题中较为常用的一种 方法。应急问题是需求推动的, 因此需求是最常用 的划分情景的依据 ${ }^{[17]}{ }^{[15]}[16]$ ，而交通是应急物流运 行的承载体也是容易产生不确定的因素 ${ }^{[20]}$ 。从本质 上而言, 应急问题不确定性来源于灾害不确定性, 因此利用灾害风险分析的结果划分情景则更加有依 据 ${ }^{[00]}[21]$ 。

灾害发展是一个连贯的影响过程并且影响因素 复杂, Barbarosoglu ${ }^{[4]}$ 根据地震的震级划分地震情景

（ES, earthquake scenario）; 然后根据每种地震情 景下的影响划分不同的影响情景 (IS, impact scenario）。王旭坪等 ${ }^{[39]}$ 利用具有不确定性的多属性 向量对应急路径每个路段未来可能的情景加以分 析。

（3）概率分布法

如果根据经验或数据估计不确定参数的近似分布, 可以用概率分布描述不确定参数, 即令 $\zeta$ 服从分布 $\Phi(\cdot)$ 。

如, Shen 等 ${ }^{[30]}$ 假设运输时间和需求点需求量是 服从对数正态分布，从而建立了大规模灾害下的两 阶段车辆路径模型; Beamon 和 Kotleba ${ }^{[42]}$ 假设需求 服从均匀分布, 建立了库存控制模型, 以确定在长 期应急响应中最优订货量和订货点; $\mathrm{He}$ 和 $\mathrm{Hu}$ 等 ${ }^{[23]}$ 则假设需求点的需求为泊松分布, 为应急供应链系 统建立了一个多重营救模型。

（4）机会约束法

在应急条件下, 要求绝对满足某些约束条件是 不现实的, 机会约束规划 (Chance constrained programming) 模型可以解决这样的问题 ${ }^{[43,44]}$, 为允 
表 1 主要文献分类汇总

\begin{tabular}{|c|c|c|c|c|c|c|c|c|c|c|c|c|c|c|c|}
\hline \multirow{2}{*}{$\begin{array}{l}\text { 不确定 } \\
\text { 性实现 } \\
\text { 方法 }\end{array}$} & \multirow{2}{*}{$\begin{array}{l}\text { 突发事 } \\
\text { 件 }\end{array}$} & \multicolumn{4}{|c|}{ 解决问题 } & \multicolumn{4}{|c|}{ 不确定性因素 } & \multicolumn{4}{|c|}{ 目标函数构成 } & \multirow[b]{2}{*}{ 求解方法 } & \multirow[b]{2}{*}{ 作者 } \\
\hline & & 选址| & 分配 & 运输 & 库存 & 需求 & 供应 & 时间 & \begin{tabular}{|l} 
其他 \\
参数
\end{tabular} & 成本 & 时间 & $\begin{array}{l}\text { 满意 } \\
\text { 度 }\end{array}$ & 其他 & & \\
\hline \multirow{4}{*}{\begin{tabular}{|l} 
区间不 \\
确定集 \\
合
\end{tabular}} & 地震 & V & $\sqrt{ }$ & & & $\sqrt{ }$ & & & & $\sqrt{ }$ & & $\sqrt{ }$ & & Lingo & 王晶等 ${ }^{[9]}$ \\
\hline & 洪水 & & $\sqrt{2}$ & & & $\sqrt{2}$ & & & & $\sqrt{2}$ & & & 末满足补偿 & Lingo & 张玲等[10] \\
\hline & 台风 & & & $\sqrt{ }$ & & $\sqrt{ }$ & & & & & & & $\begin{array}{l}\text { 受床人群暴 } \\
\text { 路度 }\end{array}$ & CPLEX & Yao 等[11] \\
\hline & 突发 & & $\sqrt{ }$ & V & & $\sqrt{ }$ & & & & $\sqrt{ }$ & & & & $\begin{array}{l}\text { 仿射调节鲁棒 } \\
\text { 优化 }\end{array}$ & Aharon 等[12] \\
\hline \multirow{14}{*}{$\begin{array}{l}\text { 情景分 } \\
\text { 析法 }\end{array}$} & 洪水 & V & $\sqrt{ }$ & & $\sqrt{ }$ & $\sqrt{ }$ & & & & $\sqrt{ }$ & & & & SAA 算法 & Chang 等 ${ }^{[13]}$ \\
\hline & 地震 & & $\sqrt{ }$ & $\sqrt{ }$ & & $\sqrt{ }$ & V & & $\begin{array}{l}\text { 道路运输 } \\
\text { 能力 }\end{array}$ & $\sqrt{ }$ & & & & GMS/OSL & Barbarosoglu \\
\hline & 突发 & V & & & & V & & & & & & & $\begin{array}{l}\text { 满足需求数 } \\
\text { 量 }\end{array}$ & CPLEX & Jia 等 ${ }^{[15]}$ \\
\hline & 地震 & V & $\sqrt{ }$ & & & $\sqrt{ }$ & $\sqrt{ }$ & & 成本 & $\sqrt{ }$ & & $\sqrt{ }$ & & 均衡规划 & $\begin{array}{l}\text { Bozorgi- } \\
\text { Amiri }^{16]}\end{array}$ \\
\hline & 风县风 & V & V & & & $\sqrt{ }$ & & & & V & & & & $\begin{array}{l}\text { 拉格朗日 } \\
\text { L-shaped 方法 }\end{array}$ & Rawls 等 ${ }^{[17]}$ \\
\hline & 地震 & V & $\sqrt{ }$ & & & $\sqrt{ }$ & & $\sqrt{ }$ & & $\sqrt{ }$ & $\sqrt{ }$ & & & GAMS/CPLEX & Mete 等 ${ }^{[18]}$ \\
\hline & 突发 & & & $\sqrt{ }$ & & & & $\sqrt{ }$ & $\begin{array}{l}\text { 安全性、 } \\
\text { 畅通度 }\end{array}$ & & & $\sqrt{ }$ & & $\begin{array}{l}\text { 蒙特卡罗模拟 } \\
\text { 和遗传算法的 } \\
\text { 混合智能方法 }\end{array}$ & 王旭坪等 ${ }^{[19]}$ \\
\hline & 突发 & & & $\sqrt{ }$ & & & & $\sqrt{ }$ & & & $\sqrt{ }$ & & & \begin{tabular}{|l} 
可靠路径搜索 \\
算法
\end{tabular} & 缪成等 ${ }^{[20]}$ \\
\hline & 突发 & & & & $\sqrt{ }$ & V & & & & & & & & GAMS/CPLEX & $\mid \begin{array}{l}\text { Salmeron 等 } \\
{[21]}\end{array}$ \\
\hline & 突发 & $\sqrt{ }$ & $V$ & & & V & $\sqrt{ }$ & & $\begin{array}{l}\text { 成本、运 } \\
\text { 输条件 }\end{array}$ & $\sqrt{ }$ & & & & $\begin{array}{l}\text { 粒子群优化算 } \\
\text { 法 }\end{array}$ & $\begin{array}{l}\text { Bozorgi- } \\
\text { Amiri 等 }\end{array}$ \\
\hline & 台风 & V & V & & & $\sqrt{ }$ & & & & & $\sqrt{1}$ & & & 遗传算法 & He 等 ${ }^{[23]}$ \\
\hline & 突发 & & & & $\sqrt{ }$ & $V$ & & & & & & & 未满足货物 & 库存模型 & Chakravarty \\
\hline & 地震 & V & V & $\sqrt{ }$ & $\sqrt{ }$ & V & & $\sqrt{ }$ & 运输成本 & $\sqrt{ }$ & & & & $\begin{array}{l}\text { CPLEX、拉格 } \\
\text { 朗日松弛算法 }\end{array}$ & Döyen $^{[25]}$ \\
\hline & 地震 & $\sqrt{ }$ & $\sqrt{ }$ & & $\sqrt{ }$ & $\sqrt{ }$ & & $\sqrt{ }$ & & $\sqrt{ }$ & & & & GAMS/CPLEX & Mete $^{[<0]}$ \\
\hline \multirow{4}{*}{ 机会约 } & 突发 & & & $\sqrt{ }$ & & & & & & & $\sqrt{ }$ & & & 混合智能算法 & 孙莉 ${ }^{[27]}$ \\
\hline & 突发 & V & & $\sqrt{ }$ & & $\sqrt{ }$ & & & & $\sqrt{ }$ & & & & CPLEX & Beraldi 等 ${ }^{[\langle 8]}$ \\
\hline & 突发 & $\sqrt{ }$ & & $\sqrt{ }$ & & $\sqrt{ }$ & & & & $\sqrt{ }$ & & & & $\begin{array}{l}\text { AIMMS } \\
\& \text { CPLEX }\end{array}$ & Beraldi 等 ${ }^{[29]}$ \\
\hline & 突发 & & $\sqrt{ }$ & $\sqrt{ }$ & & $\sqrt{ }$ & & $\sqrt{ }$ & & & $\sqrt{ }$ & & $\begin{array}{l}\text { 末满足需求 } \\
\text { 数量 }\end{array}$ & $\begin{array}{l}\text { CPLEX、禁忌 } \\
\text { 启发式 }\end{array}$ & Shen 等 ${ }^{[30]}$ \\
\hline \multirow{2}{*}{ 概率分 } & 突发 & & & V & & & & & 运输速度 & & $\sqrt{ }$ & & & $\begin{array}{l}\text { Dijkstra算法和 } \\
\text { 蚁群遗传算法 }\end{array}$ & Yuan 等 ${ }^{[31]}$ \\
\hline & 突发 & & & & $\sqrt{ }$ & $\sqrt{ }$ & & & & $\sqrt{ }$ & & & & 厍存模型 & Beamon $^{[32]}$ \\
\hline \multirow{2}{*}{\begin{tabular}{|l} 
随机过 \\
程
\end{tabular}} & 地震 & & $\sqrt{ }$ & & & V & & & & $\sqrt{ }$ & $\sqrt{ }$ & & & \begin{tabular}{|l} 
分科夫决策 \\
分析
\end{tabular} & 王炜等 ${ }^{[33]}$ \\
\hline & 突发 & & $\sqrt{ }$ & & & & & $\sqrt{ }$ & \begin{tabular}{|l|} 
灾害发生 \\
概率
\end{tabular} & V & & & 人口数量 & $\begin{array}{l}\text { 省科夫过程 } \\
\text { 分析 }\end{array}$ & $\begin{array}{l}\text { Economou 等 } \\
{[34]}\end{array}$ \\
\hline \multirow{4}{*}{ 模糊集 } & 突发 & & $\sqrt{ }$ & & & $\sqrt{ }$ & & $\sqrt{ }$ & & & & $\sqrt{ }$ & & \begin{tabular}{|l} 
基于仿真优化 \\
算法
\end{tabular} & 刘春林 ${ }^{[35]}$ \\
\hline & 地震 & & V & V & & V & & $\sqrt{ }$ & 风险 & $\sqrt{ }$ & $\sqrt{ }$ & & 风险 & 混沌优化算法 & Zheng 等 ${ }^{\mid 30]}$ \\
\hline & 突发 & & & & $\sqrt{ }$ & & & $\sqrt{ }$ & & & $\sqrt{ }$ & & & $\begin{array}{l}\text { 模拟退火算 } \\
\text { 法 }\end{array}$ & 杨勃等 ${ }^{[37]}$ \\
\hline & 突发 & & $\sqrt{ }$ & $\sqrt{ }$ & & $\sqrt{ }$ & & & 成本 & $\sqrt{ }$ & & & & 库存模型 & 钱佳 ${ }^{[36]}$ \\
\hline
\end{tabular}

许所作决策在一定程度上不满足约束条件，即约束 条件满足概率不小于某一置信水平。即令

$$
\operatorname{Pr}\left\{g_{\theta}(x, \zeta) \leq 0, \theta=1,2, \ldots p\right\} \geq \alpha
$$

其中 $g_{\theta}(x, \zeta), \theta=1,2, \ldots, p$, 为随机约束函数, 是预先设 定的置信水平。

比如, Beraldi 等 ${ }^{[28,29]}$ 在研究急救医疗服务问题 时利用机会约束条件描述服务车辆以一定概率满足 
需求量。Shen 等 ${ }^{[30]}$ 研究了大规模突发事件下的车辆 规划问题时用机会约束表示了需求和时间的不确定 性; 孙莉 ${ }^{[27}$ 对不确定环境下应急物资配送问题, 将 车辆在路径上的行走时间和运输风险设为模糊变 量, 建立了不确定环境下的机会约束规划模型。

(5) 随机过程

随机过程理论常被用于刻画具有时变特点的不 确定性。令 $X_{1}, X_{2}, X_{3}, \ldots, X_{n} \ldots$ 随机变量的一个数 列, $X_{n}$ 的值是在时间 $n$ 的状态。如果 $X_{\mathrm{n}+1}$ 对于过去 状态的条件概率分布仅是 $X_{n}$ 的一个函数, 则

$$
p\left(X_{n+1}=x \mid X_{0}, X_{1}, X_{2}, \ldots, X_{n}\right)=p\left(X_{n+1}=x \mid X_{n}\right)
$$

由于马尔科夫过程的特殊性质，在一般物流问 题中经常被采用, Chakravarthy 和 Daniel ${ }^{[45]}$ 将需求假 设为马尔科夫过程, 补货期假设为负指数分布, 并 证明供应过程也可以看作马尔科夫过程。Feeney 和 Sherbrooke ${ }^{[46]}$ 假定需求是任意复合泊松过程。在应 急过程中, 王玮等 ${ }^{[33]}$ 通过马尔科夫决策过程实现应 急资源调度方案的动态优化。Economou 和 Fakinos $^{[34]}$ 运用马尔可夫决策方法, 刻画了灾害变化 下应急资源调配的动态优化过程。

概率分布和随机过程都需要足够的历史数据积 累估计统计分布, 多适用于较常规的应急问题, 如 消防和医疗急救等。但在极端灾害的应急物流方面 还没有足够的历史数据积累, 很多情况下对于不确 定参数单纯的统计方法难以完全适用。

（6）模糊集

在巨灾的应急物流管理过程中, 通常是在缺乏 数据经验和信息条件下，面对复杂问题要运转一个 复杂系统。有很多难以定量的指标, 它们没有分明 的数量界限。模糊数是一个解决定性指标有效手段

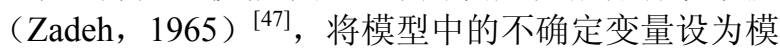
糊变量 $\xi$, 其对应的模糊集为 $\Xi$, 然后用 $\mu_{g}(\xi)$ 来 表示模型中该因素对应的隶属函数。模糊隶属度的 形式有很多种, 比如三角模糊函数、梯形模糊函 数、区间函数等。

Zheng 和 Ling ${ }^{[36]}$ 把公路运输时间、需求量、救 灾物资的期望到达时间和预计到达时间、购置额外 物资的上限、价格等不确定变量设为三角模糊变 量。刘春林 ${ }^{[3]}$ 利用拟梯形模糊隶属度关系定义应急 供应时间满意度和数量满意度。杨勃 ${ }^{[37]}$ 采用模糊数 来描述出救点到受灾点的时间。汪传旭和邓先明

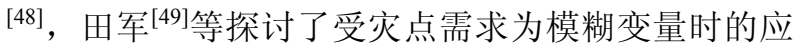
急救援车辆路径与物资运输优化问题。Behret 和 Kahraman ${ }^{[50]}$ 将需求、存货、短缺成本看作一个三角
形隶属函数模糊集并且随时间模糊集的模糊性降 低, 预测越来越准确, 具有时间动态性。

（7）函数表达法

在应急响应过程中, 由于灾后影响, 有一些因 素会随着时间或其他连续因素的变化而变化。即用 $\xi(t)$ 表示随时间变化的不确定因素。函数表达法最 常用于描述应急交通中交通流量变化状态，因为交 通流问题已经有了较完备的研究基础。但在其他方 面函数建立需要大量数据和精确的物理过程描述。 如, Yuan 等 ${ }^{[31]}$ 考虑到随着灾害发生后的时间和影响 范围的扩大, 运输速度是不确定的。因此引入速度 随时间连续变化的函数来描述速度的不确定性.

$$
v_{\mathrm{hm}}(t)=v_{\mathrm{hm}}^{0} \times \alpha_{\mathrm{hm}} \times \mathrm{e}^{-\beta_{\mathrm{hm}} \mathrm{t}}
$$

其中, $v_{\mathrm{hm}}(t)$ 为灾害条件下 $t$ 时刻弧段 $(\mathrm{h}, \mathrm{m})$ 上的 速度； $v_{\mathrm{hm}}^{0}$ 为正常条件下弧段 $(\mathrm{h}, \mathrm{m})$ 上的速度; $\alpha_{\mathrm{hm}}$ 和 $\beta_{\mathrm{hm}}$ 为在弧段 $(\mathrm{h}, \mathrm{m})$ 的参数, 根据弧段到灾害中心 的距离推断出来。

Fiedrich 等人 ${ }^{[51]}$ 利用指数函数描述了受伤人数随 时间变化的规律, 建立了动态规划模型, 解决地震 灾害发生后的应急资源配置问题。

以上几种方法有时并不是单独使用的，可以多 种方法结合起来。如, 孙莉 ${ }^{[30]}$ 对不确定环境下应急 物资配送问题进行研究, 根据实际情况将车辆在路 径上的行走时间和运输风险设为模糊变量, 建立了 不确定环境下的机会约束规划模型; Beraldi 等 ${ }^{[29]}$ 针 对了在应急系统中的选址问题, 用不同的情景来描 述需求的变化, 建立了两阶段的机会约束规划模 型; Shen 等 ${ }^{[30]}$ 研究了大规模突发事件下的车辆规划 问题并根据实际情况将问题分为两个阶段一规划阶 段和运营阶段，假设运输时间和需求点的需求量是 服从对数正态分布的变量, 建立了两阶段的机会约 束规划模型。

\section{2 不确定需求预测}

在传统物流中, 供应商和零售商对于商品的历 史信息记录较为完备, 市场表现较为稳定, 时间序 列分析技术作为一项比较灵活的动态分析模型被广 泛应用, 但这些模型比较适用于较长时间的需求预 测和随机规律刻画。在应急物流决策中, 准确的需 求信息是保障和驱动所有决策正确实施的前提。应 急响应的大部分活动都是在紧迫、随时变化甚至是 通讯不畅通或中断的情况下进行的, 需求的预测只 能根据灾前风险评估结果和应急状态下受灾点的实 时信息进行。 
在灾害发生之前, 可以根据灾害影响和区域社 会经济特征估算需求, 如聂高众等 ${ }^{[52]}$ 提出了基于地 区和季节系数城市灾害事故救援力量需求量的预测 模型。王楠等 ${ }^{[3]}$ 选取受灾人口、直接经济损失、受 灾面积、灾害强度等 4 个因素, 建立了他们与救援物 资需求的回归预测模型。这种预测模型适用于可预 报的灾害或者为了应急储备做出粗略预估, 精确度 较低。

而在灾害发生之后, 可能无法及时获取需求信 息, 即使可以获取, 需求信息来源混杂, 各级政 府、灾民自身、企业和 $\mathrm{NGO}$ 等等各类组织都会发布 需求信息, 针对这种情况, Sheu ${ }^{[54]}$ 应用信息熵模型 计算每个发布信息的权重, 进而用熵权法确定预测 动态救助需求。这个方法在目前信息传播渠道多样 化情况下是值得借鉴的。

对于具有时变特点的不确定需求, 贝叶斯更新 是广泛应用于传统物流的方法 ${ }^{[55-58]}$ 。在应急响应 中, 通过灾情上报制度、无人机侦查、遥感等手 段, 可以有效获取相关附加信息。因此可以利用贝 叶斯更新方法更加准确预测需求。如 Lodree 和

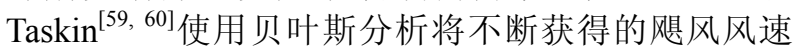
信息融合到应对县风的物资储备决策中，建立了一 个修正的报童库存模型。Choi 等 ${ }^{[5]}$ 比较了两类贝叶 斯更新模型, 预测季节性产品的均值和方差先验概 率。詹沙否等 ${ }^{[61]}$ 利用贝叶斯定理, 对灾害情景的发 生概率进行更新和修正。

\section{3 不确定优化决策目标}

应急物流问题决策往往不是以成本或收益为目 标, 而是考虑受灾公众的救助情况, 如最小化伤亡 人数 (Fiedrich $\left.{ }^{[51]}\right)$, 最小化物资到达或延迟时间 （Yuan 等 ${ }^{[31]} 、$ Zheng 等 ${ }^{[36]}$ 、Mete 等 ${ }^{[18]}$ 、孙莉 ${ }^{[27]}$ 、 缪成等 ${ }^{[20]} 、 \mathrm{He}$ 等 ${ }^{[23]}$ ), 最大化受灾群众的满意度

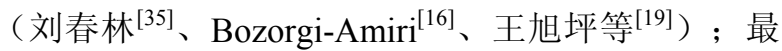
小化未满足需求物资（Shen 等 ${ }^{[30]}$ 、 Jia 等 ${ }^{[15]}$ 、 Chakravarty ${ }^{[24]}$ )。应急物流往往是一个复杂系统, 要兼顾时间、费用、损失等各方面目标, 因此很多 学者构建多目标函数 ${ }^{[16,27,62-65]}$ 作为决策目标。此外 应急问题设计到民生, 还要考虑公平性 ${ }^{[64]}$ 和均衡性 [63]。

除了应急物流问题决策目标的内容, 如何选择 决策目标的度量也非常关键。不确定优化通常采用 期望值准则, 无法计算解析解时可以采取样本均值 逼近法 (SAA： Sample Average Approximation Method) [66]。
但基于期望值准则的优化无法体现决策者的偏 好, 并且应急救助追求目标不仅是传统决策优化意 义上找到一个最高效的决策方案, 更重要的是要兼 顾这个决策方案的稳健性, 让其在极端的干扰下依 然可以比较稳定持续的运行。鲁棒优化 (Mulvey 等 ${ }^{[67]}$ ) 是一个可行选择, 其目的是找到一个近似最优 解, 使它对任意的不确定性参数观测值不敏感。目 前鲁棒优化主要采取两种处理方式, 针对使用离散 情景描述不确定问题 ${ }^{[68-73]}$, 用遗憾值或惩罚函数控 制在不同情景下最优目标值差距。针对连续的区间 不确定集合来描述不确定因素的问题 ${ }^{[9-12,74]}$ 。通常将 不确定因素转化为不确定集合的鲁棒对应问题。

突发事件的发生尤其是极端事件很多是高损失 小概率事件, 在计算过程中会被忽略。Haimes ${ }^{[75]}$ 提 出分割多目标风险法（The Partitioned Multi-objective Risk Method, PMRM），该方法引入条件期望函 数, 假定损害落入在一个特定的超越概率范围内。 这种方法可以在解决小概率事件风险度量问题, 也 可以考虑将其纳入应急问题的优化目标中。

\section{3. 主要挑战和研究展望}

应急物流问题不仅相较于传统物流问题多了不 确定的因素, 几个本质性的理论问题亟待解决:

应急物流运作过程刻画：实际的应急物流过程 是一个涉及政府部门、慈善组织、企业和个人等多 个节点和层级的供应体系。对应急物流的运作过程 而言, 应急供应链不是商业利益驱动, 而是纯粹的 需求拉动, 并且运作的目的不是为了满足商业利益 最大化, 而是尽量使需求满足程度增加。应急供应 链节点之间的物资流通不是完全按照契约关系，而 且不同供应方的信息不完全透明。信息的传递也不 是按照层级的关系逐级传递, 因此对于应急物流乃 至应急供应链的运作模式还需要改变传统商业物流 问题的模式，从其供需关系的角度进行重新刻画。

确定不确定因素的来源: 多数研究关注应急物 流不确定刻画, 如果要从根本上解决不确定性问 题, 需要从在应急物流运作分析基础上, 探讨应急 物流中信息与物资的传递变化规律, 进而发现信息 不确定的根源。此外, 由社会與论导向、信息传播 等因素产生的不确定性定量刻画与分析还相对薄 弱。目前无人机、遥感等技术手段可以用于灾情损 失核查, 这些实时信息的更新对应急物流运作不确 定性条件的影响也需要充分考虑。

应急过程风险评估：应急物流决策活动以减轻 灾害产生的风险后果为目标, 因此在自然灾害风险 评估体系中应纳入应急物流过程的风险要素。灾害 
风险评估理论框架包括致灾因子危险性与承灾体脆 弱性评估两部分, 多数是以损失评估为结果, 并不 涉及与减轻灾害风险相关的决策活动, 导致风险决 策与风险评估脱节。显而易见, 应急响应各个环节 的决策活动对于灾害风险最终结果是有影响的, 但 这种动态决策活动如何影响风险结果还没有理论指 导。如果将风险评估应用于指导决策，必须要将应 急过程风险内容加入到风险评估环节。

\section{参考文献}

[1] Sheu J B. An emergency logistics distribution approach for quick response to urgent relief demand in disasters. Transportation Research Part E: Logistics and Transportation Review, 2007, 43(6): 687-709.

[2] Yi W, Kumar A. Ant colony optimization for disaster relief operations. Transportation Research Part E: Logistics and Transportation Review, 2007, 43(6): 660-672.

[3] Kovács G, Spens K M. Humanitarian logistics in disaster relief operations. International Journal of Physical Distribution and Logistics Management, 2007, 37(2): 99114.

[4] 陈丽群. 应急物流中物资调运研究综述. 物流科技, 2008, (12): 4-6.

Chen L Q. Renew on the research of material distribution in emergency logistics . Logistics Sci-tech, 2008, 31(12):4-6.

[5] 李创. 国内外应急物流研究综述. 华东经济管理, 2013, (06): 160-165.

Li C. Review on domestic and foreign research on emergency logistics. East China Economic Management, 2013, (06): 160-165.

[6] 高文军, 陈菊红, 胡飞虎. 我国应急物流研究综述与展望. 物流科技, 2009, (08): 6-10.

Gao W J, Chen J H, Hu F H et al.The retrospect and prospect of chinese emergency logistics study. Logistics Scitech,2009,32(8):6-10.

[7] Galindo G, Batta R. Review of recent developments in OR/MS research in disaster operations management. European Journal of Operational Research, 2013, 230(2): 201-211.

[8] News B. Counting disaster: Who's dying where? http://www.bbc.co.uk/news/science-environment15592761 . 2011-11-05.

[9] 王晶, 张玲, 黄钧, 等. 基于不确定需求的鲁棒应急物流 系统. 数学的实践与认识, 2009, 39(20): 53-60.

Wang J, Zhang L, Huang J. The robust emergency logistics system based on uncertain demand Mathematics in Prantice and Theory, 2009, 39(20):53-60.

[10] 张玲, 王晶, 黄钧. 不确定需求下应急资源配置的鲁棒优 化方法. 系统科学与数学, 2010, 30(10): 1283-1292.

Zhang L, Wang J, Huang J. Robust optimal resource allocation model for uncertain demands.. Journal of Systems Science and Mathematical Sciences, 2010, 30(10): 1283-1292.
[11] Yao T, Mandala S R, Chung B D. Evacuation Transportation Planning Under Uncertainty: A Robust Optimization Approach. Networks \& Spatial Economics, 2009, 9(2): 171-189.

[12] Ben-Tal A, Chung B D, Mandala S R, et al. Robust optimization for emergency logistics planning: Risk mitigation in humanitarian relief supply chains. Transportation Research Part B Methodological, 2011, 45(8): 1177-1189.

[13] Chang M S, Tseng Y L, Chen J W. A scenario planning approach for the flood emergency logistics preparation problem under uncertainty. Transportation Research Part E Logistics \& Transportation Review, 2012, 43(6): 737-754.

[14] Barbarosoğlu G, Arda Y. A two-stage stochastic programming framework for transportation planning in disaster response. Journal of the Operational Research Society, 2004, volume 55(1): 43-53(11).

[15] Jia H, Ordóñez F, Dessouky M. A modeling framework for facility location of medical services for large-scale emergencies. Iie Transactions, 2007, 39(1): 41-55.

[16] Bozorgi-Amiri A, Jabalameli M S, Al-E-Hashem S M J M. A multi-objective robust stochastic programming model for disaster relief logistics under uncertainty. Operations Research-Spektrum, 2013, 35(4): 905-933.

[17] Rawls C G, Turnquist M A. Pre-positioning of Emergency Supplies for Disaster Response// Pre-positioning of Emergency Supplies for Disaster Response. IEEE International Symposium on Technology and Society. 1-9.

[18] Mete H O, Zabinsky Z B. Stochastic optimization of medical supply location and distribution in disaster management. International Journal of Production Economics, 2010, 126(1): 76-84.

[19] 王旭坪, 李小龙, 郭武斌. 基于情景分析的应急路径选择 研究. 运筹与管理, 2012, (5): 67-72.

Wang X P, Li X L, Guo W B, et al. Emergency path selection based on scenario analysis . Operations Research and Management Science, 2012,(5):67-72.

[20] 缪成, 吴启迪, 许维胜. 突发灾害下可靠路径搜索模型与 算法. 计算机工程与应用, 2007, 43(28): 1-3.

Miao C, Wu Q D, Xu W S .Model and algorithm of reliable path finding under sudden-onset disaster. Computer Engineering and Applications, 2007, 43(28):1-3.

[21] Salmerón J, Apte A. Stochastic Optimization for Natural Disaster Asset Prepositioning. Production \& Operations Management, 2010, 19(5): 561-574.

[22] Bozorgi-Amiri A, Jabalameli M S, Alinaghian M, et al. A modified particle swarm optimization for disaster relief logistics under uncertain environment. International Journal of Advanced Manufacturing Technology, 2011, 60(1-4): 357-371.

[23] Xinhua He W H. Modeling Relief Demands in an Emergency Supply Chain System under Large-Scale Disasters Based on a Queuing Network. Scientific World Journal, 2014, 2014(1): 195053-195053. 
[24] Chakravarty A K. Humanitarian relief chain: Rapid response under uncertainty. International Journal of Production Economics, 2014, 151(151): 146-157.

[25] Döyen A, Aras N, Barbarosoğlu G. A two-echelon stochastic facility location model for humanitarian relief logistics. Optimization Letters, 2011, 6(6): 1123-1145.

[26] Mete H O, Zabinsky Z B. Preparing for disasters: medical supply location and distribution.

[27] 孙莉. 不确定环境下应急物资配送优化研究. 兰州交通大 学, 2013.

[28] Beraldi P, Bruni M E, Conforti D. Designing robust emergency medical service via stochastic programming. European Journal of Operational Research, 2004, 158(1): 183-193.

[29] Beraldi P, Bruni M E. A probabilistic model applied to emergency service vehicle location. European Journal of Operational Research, 2009, 196(1): 323-331.

[30] Shen Z, Dessouky M, Ordóñez F. Stochastic vehicle routing problem for large-scale emergencies. Ise Working Paper, 2007.

[31] Yuan Y, Wang D. Path selection model and algorithm for emergency logistics management. Computers \& Industrial Engineering, 2009, 56(3): 1081-1094.

[32] Beamon B M, Kotleba S A. Inventory modelling for complex emergencies in humanitarian relief operations. International Journal of Logistics, 2006, 9(1): 1-18.

[33] 王炜, 刘茂, 王丽. 基于马尔科夫决策过程的应急资源调 度方案的动态优化. 南开大学学报:自然科学版, 2010, (3) 18-23.

W W, Liu M, Wang L. The dynamic optimal method of emergency resources deployment planning based on Markov decision process. Acta Scientiarum Naturalium Universitatis Nankaiensis. 2010, (3): 18-23.

[34] Economou A, Fakinos D. A continuous-time Markov chain under the influence of a regulating point process and applications in stochastic models with catastrophes. European Journal of Operational Research, 2003, 149(3): 625-640.

[35] 刘春林, 施建军, 李春雨. 模糊应急系统组合优化方案选 择问题的研究. 管理工程学报, 2002, 16(2): 25-28.

Liu C L,Shi J J,Li C Y,et al.Selection of the combinatorial optimal scheme for fuzzy emergency system. Journal of Industrial Engineering and Engineering Management, 2002, 16(2):25-28.

[36] Zheng Y J, Ling H F. Emergency transportation planning in disaster relief supply chain management: a cooperative fuzzy optimization approach. Soft Computing, 2013, 17(7): 1301-1314.

[37] 杨勃, 李小林, 杜冰. 模糊环境下应急系统多目标调度问 题求解. 系统管理学报, 2013, 22(4): 518-525.

Yang B,Li X L,Du B,et al. Solving Multi-objective emergency scheduling problem in fuzzy environments. Journal of Systems \& Management, 2013, 22(4):518-525.

[38] 钱佳. 不确定环境下模糊应急物资库存模型. 上海海事大 学学报, 2010, 31(1): 33-38.
Qian J. Fuzzy inventory models of emergency supplies under uncertain environment. Journal of Shanghai Maritime University, 2013, 22(4):518-525.

[39] 王旭坪, 李小龙, 郭武斌. 基于情景分析的应急路径选择 研究. 运筹与管理, 2012, (5): 67-72.

Li X L, Li X L, Guo W B. Emergency path selection based on scenario analysis. Operations Research and Management Science, 2012, (5): 67-72.

[40] Chang M S, Tseng Y L, Chen J W. A scenario planning approach for the flood emergency logistics preparation problem under uncertainty. Transportation Research Part E: Logistics and Transportation Review, 2007, 43(6): 737-754.

[41] Barbarosolu G, Arda Y. A two-stage stochastic programming framework for transportation planning in disaster response. Journal of the Operational Research Society, 2004, 55(1): 43-53.

[42] Kotleba B M B, A. S. Inventory modelling for complex emergencies in humanitarian relief operations. International Journal of Logistics, 2006, 9(1): 1-18.

[43] 张英楠, 牟德一, 李辉. 基于机会约束规划的航班应急调 度问题研究. 中国安全科学学报, 2012, 22(12): 82 .

Zhang Y N, Mou D Y, Li H ,et al.Solving flight emergency scheduling problem based on chance-constrained programming. China Safety Science Journal, 2012, 22(12):82-88.

[44] Charnes A, Cooper W W. Chance-Constrained Programming. Management Science, 1959, 6(1): 73-79.

[45] Chakravarthy S R, Daniel J K. A Markovian inventory system with random shelf time and back orders. Computers \& Industrial Engineering, 2004, 47(4): 315-337.

[46] Feeney G J, Sherbrooke C C. The S-1, S Inventory Policy Under Compound Poisson Demand. Manage Sci, 1966, 12(5): 391-411.

[47] Zadeh L A. Fuzzy sets . Information \& Control, 1965, 8(65): 338-353.

[48] 汪传旭, 邓先明. 模糊环境下多出救点应急救援车辆路径 与物资运输优化研究. 系统管理学报, 2011, 20(3): 269275.

Wanf C X, Deng X M. Multi-depot emergency vehicle routing and transportation optimization with fuzzy variables Journal of Systems \& Management, 2011, 20(3): 269-275.

[49] 田军, 马文正, 汪应洛,等. 应急物资配送动态调度的粒子 群算法. 系统工程理论与实践, 2011, 31(5): 898-906.

Tian J, Ma W Z, Wang Y L, et al. Emergency supplies distributing and vehicle routes programming based on particle swarm optimization. Systems Engineering - Theory \& Practice, 2011, 31(5): 898-906.

[50] Behret H, Kahraman C. A multi - period newsvendor problem with pre - season extension under fuzzy demand. Journal of Business Economics \& Management, 2010, 11(4): 17.

[51] Fiedrich F, Gehbauer F, Rickers U. Optimized resource allocation for emergency response after earthquake disasters. Safety Science, 2000, 35(1-3): 41-57. 
[52] 聂高众, 高建国, 苏桂武,等. 地震应急救助需求的模型化 处理一来自地震震例的经验分析. 资源科学, 2001, 23(1): 69-76.

Nie G Z, Gao J G, Su G W, et al. Models on rapid judgment for the emergent rescue needs during earthquake-by analysis on post-earthquake events.Resources Science,2001, 1, 23(1): 69-76.

[53] 王楠, 刘勇, 曾敏刚. 自然灾害应急物流的物资分配策略 研究. 第五届中国物流学术年会, 2006.

[54] Sheu J B. Dynamic relief-demand management for emergency logistics operations under large-scale disasters. Transportation Research Part E Logistics \& Transportation Review, 2010, 46(1): 1-17.

[55] Choi T M, Li D, Yan H. Quick response policy with Bayesian information updates. European Journal of Operational Research, 2006, 170(3): 788-808.

[56] Choi T M, Li D, Yan H. Optimal single ordering policy with multiple delivery modes and Bayesian information updates. Computers \& Operations Research, 2004, 31(12): 1965-1984.

[57] Iyer A V, Bergen M E. Quick response in manufacturerretailer channels. Management Science, 1997, 43(4): 559570.

[58] Eppen G D, Iyer A V. Improved fashion buying with bayesian updates. Operations Research, 1997, 45(6): 805819.

[59] Taskin S, Lodree E J. Inventory Decisions for Emergency Supplies Based on Hurricane Count Predictions. International Journal of Production Economics, 2010, 126(1): 66-75.

[60] Lodree E J, Taskin S. Supply chain planning for hurricane response with wind speed information updates. Computers \& Operations Research, 2009, 36(1): 2-15.

[61] 詹沙砧, 刘南. 基于灾情信息更新的应急物资配送多目标 随机规划模型. 系统工程理论与实践, 2013, 33(1): 159166.

Zhan S L, Liu N. Multi-objective stochastic programming model for relief allocation based on disaster scenario information updates. Systems Engineering - Theory \& Practice, 2013, 33(1): 159-166.

[62] Gong Q, Batta R. Allocation and reallocation of ambulances to casualty clusters in a disaster relief operation. Iie Transactions, 2007, 39(1): 27-39.

[63] 朱建明. 损毁情景下应急设施选址的多目标决策方法. 系 统工程理论与实践, 2015, (3): 720-727.

Zhu J M. Methods of multi-objective decision-making for emergency facility location problem under failure scenario. Systems Engineering - Theory \& Practice, 2015, (3): 720727.

[64] Huang M, Smilowitz K, Balcik B. Models for Relief Routing: Equity, Efficiency and Efficacy. Procedia - Social and Behavioral Sciences, 2011, 17(1): 416-437.
[65] Zhang Z H, Jiang H. A robust counterpart approach to the bi-objective emergency medical service design problem. Applied Mathematical Modelling, 2014, 38(3): 1033-1040.

[66] Pagnoncelli B K, Ahmed S, Shapiro A. Sample Average Approximation Method. Mat Puc.

[67] Mulvey J M, Zenios S A. Robust optimization of largescale systems. Operations Research, 1995, 43(2): 264-281.

[68] 刘波, 李波, 李砚. 不确定条件下应急资源布局的鲁棒双 层优化模型. 计算机工程与应用, 2013, 49(16): 13-17.

Liu B, Li B, Li Y. Robust bi-level optimization model for emergency resource location and allocation under uncertain condition .Computer Engineering and Applications, 2013,(16):13-17.

[69] 姜涛, 朱金福. 应急设施鲁棒优化选址模型及算法. 交通 运输工程学报, 2007, 7(5): 101-105.

Jiang T, Zhu J F. Robust optimization model and algorithm of emergency establishment .Journal of Traffic and Transportation Engineering, 2007, 7(5):101-105.

[70] 王保华, 何世伟. 不确定环境下物流中心选址鲁棒优化模 型及其算法. 交通运输系统工程与信息, 2009, 9(2): 69-74. Wang B H, He S W. Robust optimization model and algorithm for logistics center location and allocation under uncertain environment. Journal of Transportation Systems Engineering and Information Technology, 2009, 9(2): 69-74

[71] 钟慧玲, 庄楠, 张冠湘, 等. a-鲁棒的危险品道路运输应 急设施选址问题. 系统工程理论与实践, 2013, 33(5): 1262-1268

Zhong $\mathrm{H} \mathrm{L}$, Zhuang $\mathrm{N}$, Zhang $\mathrm{G} \mathrm{X}$, et al. a -robust emergency facilities location problems for hazardous materials transportation. Systems Engineering - Theory \& Practice, 2013, 33(5): 1262-1268.

[72] 朱雷, 黎建强, 汪明. 不确定条件下应急管理人力供应链 多功能资源配置鲁棒优化问题. 系统工程理论与实践, 2015, (3): 736-742.

Zhu L, Li J Q, Wang M. Multi-resource robust optimization of emergency human resource supply chain management under uncertainty. Systems Engineering - Theory \& Practice, 2015, (3): 736-742.

[73] 陈涛, 黄钧, 朱建明. 基于信息更新的两阶段鲁棒-随机优 化调配模型研究. 中国管理科学, 2015, 23(10): 67-77.

Chen T, Huang J, Zhu J M. Two-stage Robust-Stochastic Decision Model for Relief Allocation Based on Disaster Scenario Information Updata. Chinese Journal of Management Science, 2015, 23(10): 67-77.

[74] Yao T, Bental A, Chung B D, et al. Robust Optimization for Dynamic Traffic Assignment Under Demand Uncertainty// Robust Optimization for Dynamic Traffic Assignment Under Demand Uncertainty. Transportation Research Board 89th Annual Meeting, 2010.

[75] 海姆斯. 风险建模、评估和管理. 西安: 西安交通大学出 版社, 2007. 